\title{
Association of Foster Care and its Duration with Clinical Symptoms and Impairment: Foster Care versus Non-Foster Care Comparisons with Spanish Children
}

\author{
Ignasi Navarro-Soria $\mathbb{( i b}^{1} \cdot$ Mateu Servera ${ }^{2} \cdot$ G. Leonard Burns ${ }^{3}$
}

(c) The Author(s) 2019

\begin{abstract}
Objective The objective was to determine if Spanish foster care children and Spanish non-foster children differ on sluggish cognitive tempo (SCT), ADHD-inattention (IN), ADHD-hyperactivity/impulsivity (HI), oppositional defiant disorder (ODD), limited prosocial emotions (LPE), anxiety, depression, social and academic impairment measures and if the duration of foster care predicts a reduction in symptom and impairment differences between foster and non-foster care children. Method Foster care parents of 49 children ( 8 to 13 years, 57\% girls) and non-foster care mothers and fathers of 1776 children ( 8 to 13 years, $49 \%$ girls) completed the Child and Adolescent Behavior Inventory (CABI).

Results Foster care children had significantly higher scores on all symptom and impairment measures than non-foster care mother and father groups ( $d$ values from 0.37 to 1.53). A longer duration in foster care (range 8 to 86 months) was also associated with significant lower scores on SCT, ADHD-IN, anxiety, depression, and academic impairment. In addition, while foster care children with a shorter duration in foster care (less than medium months) had significantly higher scores than the non-foster care groups on all measures ( $d$ values from 0.66 to 2.25), children with a longer duration in foster care did not differ from the non-foster care groups on anxiety, depression, ADHD-IN, ADHD-HI, LPE, social and academic impairment.

Conclusions Although foster care children had elevated psychopathology and impairment scores relative to non-foster care children, a longer stay in foster care was associated with the elimination of the difference on most symptom and impairment measures.
\end{abstract}

Keywords Foster care $\cdot$ Protection system $\cdot$ Risk of social exclusion $\cdot$ Abandonment $\cdot$ Clinical picture

UNICEF reports that approximately 8 million children worldwide live in institutions (Pinheiro 2006). Numerous studies have demonstrated that residential foster care can have negative consequences for children (e.g., Baptista et al. 2014; Delgado et al. 2012; Dregan and Gulliford 2012; Li et al. 2017; López et al. 2010; Rutter et al. 2010; Smyke et al. 2012; Troller-Renfree et al. 2018; Tibu et al. 2014). The findings from these various studies resulted in recommendations to limit residential foster care and to promote

\footnotetext{
Ignasi Navarro-Soria

ignasi.navarro@ua.es

1 University of Alicante, San Vicente, Spain

2 University of the Balearic Islands, Palma de Mallorca, Spain

3 Washington State University, Pullman, USA
}

home-based foster care (Tarren-Sweeney 2017), especially for young children (Dozier et al. 2012; Palacios 2003; Del Valleand Bravo 2003; Del Valle et al. 2008). However, while there is considerable knowledge about the negative consequences of residential foster care, there is less knowledge on the impact of home-based foster care.

Foster care, already introduced in the 1987 law that created a new child protection system in Spain, was proposed as the great alternative for those children who had to be separated from their families. Up until then, the solution used in most cases was the admission to a center or foster home, were they remained for many years, if not all of their childhood until reaching coming of age (Del Valle et al. 2009). The subsequent 1/96 Organic Law regarding the Legal Protection of Minors, established as a basic principle the upbringing of the child in a family context, either by recuperating the functions of good parenting by the families 
(with family intervention programs and support materials), or by the fostering of the child by another family or even adoption (López et al. 2010). The priority that children always have the chance of developing within a family is one of the issues of greater legal, scientific and profesional consensus. However, in the thirty years that have elapsed, family foster care has not become a priority measure and residential care remains the predominant solution (Del Valle et al. 2009).

The report of EUROARRCC (1998) offers an overview of the characteristics of children in home-based foster care. With foster care samples from Finland, Scotland, Ireland, and Spain, the findings indicated that $67 \%$ of the children had scores in the clinical range on internalizing and externalizing symptom dimensions. In a more recent study with Australian children in foster care (Sawyer et al. 2007), 61\% of the children were in the clinical range on the internalizing and externalizing symptom dimensions (care-giver ratings on the CBCL). Similar results have occurred in other studies (e.g., Goemans et al. 2018; Humphreys et al. (2015). These studies suggest that many of the children in home-based foster care have scores in the clinical range on various symptom and impairment dimensions.

Although it is clear that many children in home-based foster care have scores in the clinical range, only a few studies have examined the psychological problems of children in home-based foster care in Spain. A study by Gil-Lario et al. (2013), for example, evaluated 32 children in foster care with BASC ratings by foster parents and teachers. Foster parents rated the children as significantly higher on internalizing, attention, and externalizing symptom dimensions as well as significantly lower on social and adaptive skills than teachers. In addition, mean scores on the BASC scales for the 32 children were in the clinical range for the attention (parents and teachers), externalizing (foster parents only), and adaptive skills scales (foster parents only). This study, however, did not include a comparison group and also did not evaluate if the duration of time in home-based foster care was associated with improvements in the symptom and impairment scores.

Another Spanish study was undertaken by FernándezMolina et al. (2011), in order to measure behavioral problems in adolescents who were in pre-adoptive, residential and extended family care, using the Child Behavior Checklist (CBCL). These researchers found that adolescents in residential care had higher scores than those adolescents who are in pre-adoptive or extended family care. The researchers also concluded that adolescents in pre-adoptive care present fewer behavioral problems and are to a greater extent within the normal range of the CBCL.

The overall purpose of the study was to compare a sample of Spanish children in foster care to a non-foster care sample of Spanish children on clinical symptoms (i.e., anxiety, depression, sluggish cognitive tempo (SCT), ADHD-inattention (IN), ADHD-hyperactivity/impulsivity (HI), oppositional defiant disorder [ODD], and limited prosocial emotions [LPE]) and impairment (i.e., academic and social impairment) dimensions. The first objective was to determine the mean differences (Cohen's $d$ values) between foster care and non-foster care groups on the symptom and impairment dimensions. Given the risk factors associated with the removal of a child from the family and placement in foster care, it was expected that the foster care group would have significantly higher scores on all the symptom and impairment measures than the non-foster care group. The second objective was to determine if the foster care and non-foster care differences on the symptom and impairment dimensions remained significant after controlling for children's sex and age. It was expected that foster care children would continue to show significantly higher scores on the symptom and impairment dimensions than the non-foster care children even after controlling for sex and age. The third objective was to determine if the number of months in foster care would be associated with an improvement in the symptom and impairment dimension scores. It was expected that longer stays in foster care would be associated with lower scores on the symptom and impairment dimensions. The fourth objective was to determine if children with longer stays in foster care (greater than the medium number of months) would have symptom and impairment scores that did not differ significantly from the non-foster care group while children with shorter stays in foster care (less than the medium number of months) would still differ significantly from the non-foster care group. Answers to the questions associated with the four objectives will allow a better understanding of the mental health of Spanish children in foster care and the impact of foster care on their mental health.

\section{Methods}

\section{Participants}

\section{Foster care group}

There were 281 children in foster care at the time of the study in the Province of Alicante, Spain. A total of 87 foster care families agreed to participate in the study with 49 of these families having a foster child in the age range of 8 to 13 years of age, the age range of the non-foster care comparison sample. The mean age of the 49 children was 10.06 $(S D=1.51,57 \%$ girls). The ethnicity of the 49 children was $51 \%$ Caucasian, $32 \%$ Roma, $13 \%$ the sub-Saharan, and $4 \%$ other ethnic groups. The socioeconomic level of the foster care families was $25 \%$ medium-high income, $42 \%$ medium 
income, 24\% medium-low income, and 9\% low income with $81 \%$ two parent families and $19 \%$ single-parent families. Of the 49 families $11 \%$ reported 10 grades of education, $17 \%$ reported 12 grades of education, $26 \%$ reported vocational training (e.g., electricians, plumbers, mechanics, and administrators with three to five years of education beyond the high school diploma) and $30 \%$ reported a university degree.

\section{Comparison group}

The comparison group consisted of 1776 children from 32 elementary schools from Mallorca, Spain (see Sáez et al. 2018 for more details on this sample). The mean age of the children was 10.30 years $(S D=1.31$, range 8 to $13,49 \%$ girls). Mothers completed the rating scale on 1648 children and fathers on 1358 children.

A total of $84 \%$ of the mothers and $86 \%$ of the fathers were married with $62 \%$ of the mothers and $71 \%$ of the fathers having permanent employment (13 and 12\%, respectively, reported occasional employment). For mothers (fathers) $17 \%$ (25\%) reported 10 grades of education, $19 \%$ (22\%) reported twelve grades of education, $23 \%(21 \%)$ reported vocational training (e.g., electricians, plumbers, mechanics, and administrators with three to five years of education beyond the high school diploma) and 37\% (29\%) reported a university degree (approximately 4\% were missing).

\section{Procedures}

\section{Child and Adolescent Behavior Inventory (CABI)}

The foster care parents and the non-foster care parents completed the CABI (Burns et al. 2015a, 2015b). The foster care parents completed the CABI jointly (i.e., one CABI for each foster care child) while the non-foster care mothers and fathers completely the CABI separately. The mothers and fathers in the comparison group were instructed to complete the CABI independently. The CABI measures SCT (15 symptoms), ADHD-IN (9 symptoms), ADHD-HI (9 symptoms), ODD (8 symptoms), limited prosocial emotions (LPE, 4 symptoms of the DSM-5 with Limited Prosocial Emotions Specifier), anxiety (6 symptoms) depression (6 symptoms), social impairment (4 items: quality of interactions with parents, other adults, siblings, and peers), and academic impairment (5 items: quality of homework/classwork, reading skills, arithmetic skills, writing skills, and global academic skills).

The SCT, ADHD, ODD, LPE, anxiety, and depression symptoms were rated on a 6-point scale (i.e., almost never [never or about once per month], seldom [about once per week], sometimes [several times per week], often [about once per day], very often [several times per day], and almost always [many times per day]. A 7-point scale was used for the academic and social impairment items (i.e., severe difficulty, moderate difficulty, slight difficulty, average performance [average interactions] for grade level, slightly above average, moderately above average, and excellent performance [excellent interactions] for grade level). The LPE, academic impairment, and social impairment items were reverse keyed so that higher scores represent LPE, greater academic impairment, and greater social impairment, respectively.

Earlier studies provide support for the reliability (internal consistency, test-retest, inter-rater) and validity of scores from the ADHD-IN, ADHD-HI, ODD, LPE, anxiety, depression, social impairment, and academic impairment scales with Spanish children (e.g., Belmar et al. 2017; Bernad et al. 2016; Burns et al. 2017; Sáez et al. 2018; Seijas et al. 2018a, 2018b; Servera et al. 2016). In the current study, Cronbach's alpha (mothers, fathers) in the non-foster care group was excellent for $\operatorname{SCT}(0.93,0.92)$, ADHD-IN (0.95, 0.95), ADHD-HI (0.93, 0.93), ODD (0.92, 0.91), LPE (0.84, 0.85), anxiety (0.72, 0.75), depression $(0.87,0.84)$, social impairment $(0.90,0.91)$, and academic impairment $(0.94,0.95)$ scores. Inter-rater correlations for mothers with fathers varied from 0.66 for anxiety to 0.87 for academic impairment. For the foster care group, Cronbach's alpha for SCT, ADHD-IN, ADHD-HI, ODD, LPE, anxiety, depression, social impairment, and academic impairment were $0.96,0.97,0.97,0.95,0.87,0.85,0.92$, 0.87 , and 0.93 , respectively.

\section{Data Analyses}

The analyses used version 8.0 of the statistical software, (Muthén and Muthén 1998-2017). The robust maximum likelihood estimator was used for the analyses (MLR). The first analysis determined the mean differences on the symptom and impairment dimensions for foster care and the non-foster care groups (i.e., the foster care ratings were compared to the non-foster care mother group and the nonfoster care father group). Cohen $d$ value and its $95 \%$ confidence interval was used to determine the magnitude of the group differences on the symptom and impairment measures. The second anlaysis regressed the symptom and impairment measures on the sex of the child, the age of the child, and the group membership (i.e., foster care versus non-foster care groups). The purpose of this analysis was to determine whether group membership would uniquely predict the symptom and impairment dimensions after controlling for the sex and age of the child. The partial standardized regression coefficients for this analysis can be intepreted in the same manner as Cohen's $d$ value (i.e., the $d$ value representing the number of standard deviations the 
foster care group is higher than the non-foster care group after controlling for the age and sex of the child). The fourth analysis determined the correlation of the number of months in foster care with the symptom and impairment dimensions. The fifth analysis created two dummy coded predictors (i.e., (1) short duration of stay in foster care [less than medium number of months, coded 1] versus non-foster care comparison group [coded 0]; and (2) long duration of stay in foster care [greater than the medium number of months, coded 1] versus non-foster care comparison group [coded 0]. The symptom and impairment dimensions were regressed on these two predictors to determine if the long stay in the foster care group would no longer differ significantly from the non-foster care group on the symptom and impairment dimensions while the short stay in foster care group would still have significantly higher scores than the non-foster care group on the symptom and impairment dimensions.

\section{Results}

\section{Missing Information}

Covariance coverage was greater than $99 \%$ for all variances and covariances for ratings by mothers, fathers, and foster parents. The participants thus left very few blank items. The MLR estimator also does not delete any cases with missing information on the outcome measures.

\section{Foster Care versus Comparison Group}

Table 1 shows the results for the comparison of the foster care and non-foster care groups. The foster care group had significantly $(p<0.05)$ higher means than the non-foster care group on all the symptom and impairment scales. The weakest effects were for the social impairment and academic impairment scales (i.e., $d$ values of approximately 0.38 for social impairment and 0.60 for academic impairment). The $d$ values for the symptom dimensions varied from 0.69 for anxiety to 1.53 for SCT with most of the $d$ values for the symptom measures being greater than 1.00 (i.e., greater than 1.00 standard deviation higher than the non-foster care group). Almost identical results occurred for the comparison of the foster care group to the non-foster care mother and father groups.

Table 2 shows the results from the partial standardized regression coefficients of the regression of the symptom and impairment measures on the age and sex of the child along with group membership (foster care versus the non-foster care group). Even after controlling for sex and age, the partial standardized regression coefficients are almost identical to the Cohen $d$ values in Table 1. Since the symptom and impairment measures are standardized, the partial standardized regression coefficients can be interpreted in the same manner as Cohen $d$ (e.g., the foster care group mean score on the anxiety measure was 0.69 standard deviations higher than the non-foster care mother group after controlling for child sex and age).

\section{Relationship of Months in Foster Care with Symptom and Impairment Measures}

Table 3 shows the correlation for the number of months in foster care with the symptom and impairment measures for the 49 children in foster care. A higher number of months in foster care was associated with significantly $(p<0.01)$ lower scores on the SCT, anxiety, depression, ADHDinattention, and academic impairment measures. Months in foster care maintained a significant $(p<0.01)$ negative
Table 1 Comparison of foster care and non-foster care groups

\begin{tabular}{|c|c|c|c|c|c|c|c|c|}
\hline & \multicolumn{2}{|c|}{$\begin{array}{l}\text { Foster } \\
\text { care } \\
\text { parents }\end{array}$} & \multicolumn{2}{|c|}{$\begin{array}{l}\text { Non-foster } \\
\text { care } \\
\text { mothers }\end{array}$} & \multicolumn{4}{|c|}{ Non-foster care fathers } \\
\hline & $M$ & $S D$ & $M$ & $S D$ & $d(95 \% C I)$ & $M$ & $S D$ & $d(95 \% C I)$ \\
\hline Anxiety & 1.02 & 1.04 & 0.57 & 0.63 & $0.70(0.41,0.98)$ & 0.56 & 0.65 & $0.69(0.40,0.97)$ \\
\hline Depression & 1.05 & 1.22 & 0.32 & 0.60 & $1.18(0.89,1.46)$ & 0.27 & 0.50 & $1.44(1.15,1.73)$ \\
\hline Sluggish cognitive tempo & 1.58 & 1.36 & 0.60 & 0.70 & $1.35(1.06,1.64)$ & 0.55 & 0.64 & $1.53(1.24,1.82)$ \\
\hline ADHD-inattention & 2.37 & 1.64 & 1.15 & 1.16 & $1.04(0.75,1.33)$ & 1.08 & 1.07 & $1.18(0.90,1.47)$ \\
\hline $\begin{array}{l}\text { ADHD-hyperactivity/ } \\
\text { impulsivity }\end{array}$ & 1.91 & 1.66 & 0.95 & 1.06 & $0.88(0.59,1.17)$ & 0.94 & 1.03 & $0.91(0.63,1.20)$ \\
\hline Oppositional defiant disorder & 1.85 & 1.34 & 0.85 & 0.88 & $1.11(0.83,1.40)$ & 0.77 & 0.83 & $1.26(0.98,1.56)$ \\
\hline Limited prosocial emotions & 2.61 & 1.52 & 1.38 & 1.22 & $1.02(0.71,1.28)$ & 1.59 & 1.23 & $0.82(0.53,1.11)$ \\
\hline Social Impairment & 2.03 & 1.37 & 1.57 & 1.26 & $0.37(0.08,0.65)$ & 1.55 & 1.26 & $0.38(0.09,0.66)$ \\
\hline Academic Impairment & 3.14 & 1.92 & 2.34 & 1.48 & $0.54(0.25,0.87)$ & 2.25 & 1.46 & $0.60(0.32,0.89)$ \\
\hline
\end{tabular}

$d=$ Cohen's $d$ (i.e., the difference between the foster care and non-foster care groups in standard deviation units). The CI (confidence interval) is for the $d$ value. All differences are significant at $p<0.05$ 


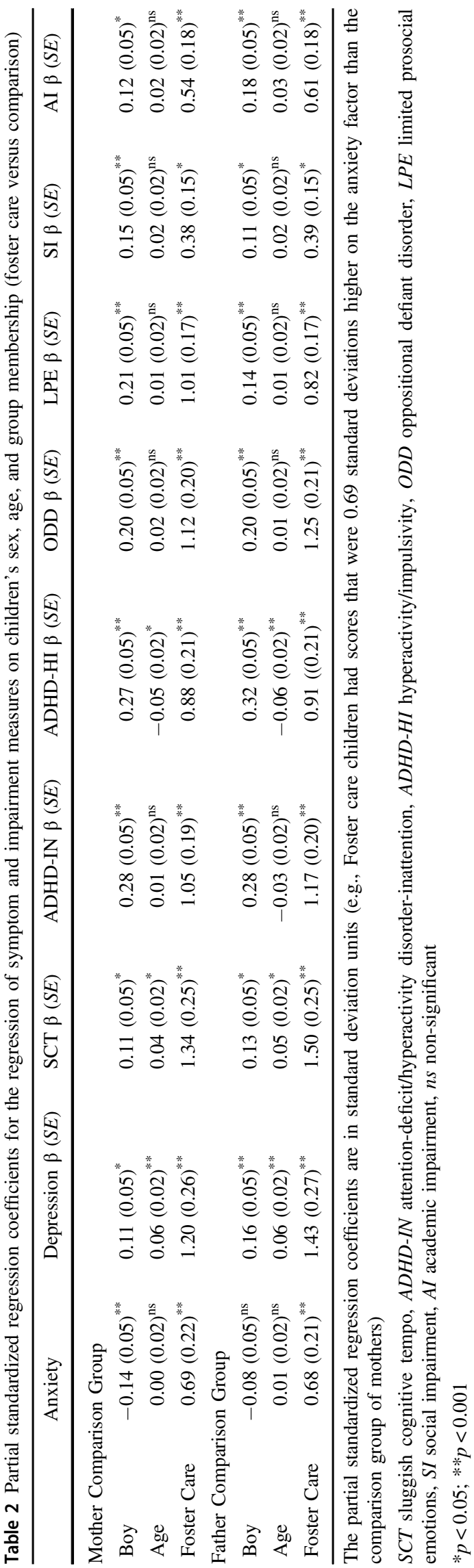

Table 3 Correlations (SEs) of number of months in foster care with symptom and impairment measures

\begin{tabular}{ll}
\hline Measures & $\begin{array}{l}\text { Months in Foster Care }(M=52.45, \\
S D=21.37, \text { range }=8 \text { to } 86)\end{array}$ \\
\hline Sluggish Cognitive Tempo & $-0.44(0.11)^{*}$ \\
Anxiety & $-0.36(0.13)^{*}$ \\
Depression & $-0.35(0.12)^{*}$ \\
ADHD-Inattention & $-0.40(0.13)^{*}$ \\
ADHD-Hyperactivity/ & $-0.16(0.11)^{\mathrm{ns}}$ \\
Impulsivity & \\
Oppositional Defiant & $-0.16(0.13)^{\mathrm{ns}}$ \\
Disorder & \\
Limited Prosocial Emotions & $-0.15(0.13)^{\mathrm{ns}}$ \\
Social Impairment & $-0.18(0.11)^{\mathrm{ns}}$ \\
Academic Impairment & $-0.32(0.11)^{*}$ \\
\hline$N=49$ & \\
$* p<0.05$ & \\
$n s$ non-significant &
\end{tabular}

association with SCT $(\beta=-0.44, S E=0.12)$, anxiety $(\beta=$ $-0.39, S E=0.12)$, depression $(\beta=-0.33, S E=0.12)$, ADHD-IN $(\beta=-0.43, S E=0.12)$, and academic impairment $(\beta=-0.32, S E=0.12)$ even after controlling for the sex and age of the children in foster care.

Table 4 shows the results from the regression of the symptom and impairment dimensions on the two dummy coded predictors (i.e., (1) short duration in foster care versus non-foster care group; (2) long duration in foster care versus non-foster care group). The children with a short duration in foster care (less than the medium number of months) had significantly $(p<0.01)$ higher scores on all the symptom and impairment dimensions than the non-foster care mother and father comparison groups. The $d$ values for these comparisons ranged from 0.66 for social impairment to 2.25 for SCT. In contrast, the children with a long duration in foster care (greater than the medium number of months) did not differ significantly $(p>0.05)$ from the non-foster care mother group on the anxiety, depression, ADHD-IN, ADHD-HI, social impairment, and academic impairment dimensions ( $d$ value for these non-significant results ranged from 0.05 to 0.48 ). Similar results occurred for the comparison of the long duration in foster care group to the non-foster care father group - that is, a lack of a significant $(p>0.05)$ difference on the anxiety, ADHD-IN, ADHD-HI, LPE, social impairment, and academic impairment dimensions ( $d$ value for these nonsignificant ranged results from 0.07 to 0.44 ).

\section{Discussion}

The purpose of our study was to compare Spanish children in foster care to Spanish children not in foster care on a 
Table 4 Partial standardized regression coefficients for the regression of symptom and impairment measures on length of stay in foster care (less than or greater than medium months) versus non-foster care mother and father groups

\begin{tabular}{|c|c|c|c|c|}
\hline \multirow{2}{*}{$\begin{array}{l}\text { Measures } \\
\text { Predictors }\end{array}$} & \multicolumn{2}{|c|}{ Mothers } & \multicolumn{2}{|l|}{ Fathers } \\
\hline & $\beta$ & $S E$ & $\beta$ & $S E$ \\
\hline \multicolumn{5}{|l|}{ Sluggish Cognitive Tempo } \\
\hline Shorter stay vs. comparison & $2.04^{* *}$ & 0.36 & $2.25^{* *}$ & 0.37 \\
\hline Longer stay vs. comparison & $0.56^{*}$ & 0.28 & $0.68^{*}$ & 0.29 \\
\hline \multicolumn{5}{|l|}{ Anxiety } \\
\hline Shorter stay vs. comparison & $1.05^{* *}$ & 0.35 & $1.02^{* *}$ & 0.33 \\
\hline Longer stay vs. comparison & $0.33^{\mathrm{ns}}$ & 0.26 & $0.33^{\mathrm{ns}}$ & 0.25 \\
\hline \multicolumn{5}{|l|}{ Depression } \\
\hline Shorter stay vs. comparison & $1.81^{* *}$ & 0.40 & $2.13^{* *}$ & 0.44 \\
\hline Longer stay vs. comparison & $0.48^{\mathrm{ns}}$ & 0.28 & $0.63^{*}$ & 0.31 \\
\hline \multicolumn{5}{|l|}{ ADHD-Inattention } \\
\hline Shorter stay vs. comparison & $1.67^{* *}$ & 0.26 & $1.85^{* *}$ & 0.28 \\
\hline Longer stay vs. comparison & $0.35^{\mathrm{ns}}$ & 0.21 & 0.44 & 0.22 \\
\hline \multicolumn{5}{|c|}{ ADHD-Hyperactivity/Impulsivity } \\
\hline Shorter stay vs. comparison & $1.37^{* *}$ & 0.32 & $1.41^{* *}$ & 0.32 \\
\hline Longer stay vs. comparison & $0.35^{\mathrm{ns}}$ & 0.24 & $0.37^{\mathrm{ns}}$ & 0.25 \\
\hline \multicolumn{5}{|l|}{ Oppositional Defiant Disorder } \\
\hline Shorter stay vs. comparison & $1.58^{* *}$ & 0.30 & $1.74^{* *}$ & 0.30 \\
\hline Longer stay vs. comparison & $0.60^{*}$ & 0.25 & $0.72^{* *}$ & 0.26 \\
\hline \multicolumn{5}{|l|}{ Limited Prosocial Emotions } \\
\hline Shorter stay vs. comparison & $1.33^{* *}$ & 0.22 & $1.15^{* *}$ & 0.22 \\
\hline Longer stay vs. comparison & $0.63^{* *}$ & 0.24 & $0.46^{\mathrm{ns}}$ & 0.24 \\
\hline \multicolumn{5}{|l|}{ Social Impairment } \\
\hline Shorter stay vs. comparison & $0.66^{* *}$ & 0.22 & $0.67^{* *}$ & 0.22 \\
\hline Longer stay vs. comparison & $0.06^{\mathrm{ns}}$ & 0.19 & $0.07^{\mathrm{ns}}$ & 0.19 \\
\hline \multicolumn{5}{|l|}{ Academic Impairment } \\
\hline Shorter stay vs. comparison & $1.00^{* *}$ & 0.24 & $1.07^{* *}$ & 0.24 \\
\hline Longer stay vs. comparison & $0.05^{\mathrm{ns}}$ & 0.23 & $0.11^{\mathrm{ns}}$ & 0.23 \\
\hline
\end{tabular}

The partial standardized regression coefficients are in standard deviation units (e.g., Shorter stay foster care children had scores that were 1.05 standard deviations higher on the anxiety factor than the comparison group of mothers). Shorter stay=less than medium number of months in foster care $(n=25)$; Longer stay $=$ greater than the medium number of months in foster care $(n=24)$

$* p<0.05 ; * * p<0.01$

$n s$ non-significant

wide range of symptom (i.e., anxiety, depression, SCT, ADHD-IN, ADHD-HI, ODD, and LPE) and impairment (social and academic) measures. More specifically, the purpose was to determine if the children in foster care had significantly higher scores on the symptom and impairment measures than the children not in foster care. In addition, we sought to determine if the number of months in foster care was associated with a decrease in the scores on the symptom and impairment measures. Finally, and perhaps the most important objective, the study sought to determine if children with longer stays in foster care (greater than the medium number of months) would have symptom and impairment scores that did not differ significantly from children not in foster care while children with shorter stays in foster care (less than the medium number of months) would still differ significantly from the non-foster care group.

The children in foster care had significant higher scores than the children not in foster care on all the symptom and impairment measures, coinciding with results from previous investigations (Goemans et al. 2018; Humphreys et al. 2015; Sawyer et al. 2007). The smallest differences ( $d$ values of 0.37 to 0.60 ) were for social and academic impairment with the differences for the symptom measures being much larger ( $d$ values from 0.69 to 1.59 ). The largest differences occurred for depression, SCT, ODD, and ADHD-IN ( $d$ values from 1.04 to 1.53). The children in foster care thus had significantly elevated scores on the symptom and impairment measures relative to the children not in foster care. In addition, a greater number of months in foster care was associated with significantly lower scores on SCT, anxiety, depression, ADHD-IN, and academic impairment measures. Finally, while children with a shorter duration in foster care (i.e., less than the medium number of months) continued to differ significantly from the children not in foster care on all the symptom and impairment measures, children with a longer duration in foster care (greater than the medium number of months) did not differ significantly from the control group on anxiety, depression, ADHD-IN, ADHD-HI, social impairment, and academic impairment. Although it is important to remember these findings are based on cross-sectional data, it was nonetheless encouraging to find such outcomes associated with the length of stay in foster care.

While our findings are consistent with a wide range of other studies that indicate that children in home-based foster care have higher clinical and impairment scores than comparison groups (Delgado et al. 2012; Dregan and Gulliford 2012; Fries et al. 2008, Gunnar and Van Dülmen 2007; Kjelsberg and Nygren 2004; Li et al. 2017; Martín et al. 2008; Shechory and Sommerfeld 2007; Smyke et al. 2012), our findings are more unique in the outcome that children with longer stays in foster care did not differ from the nonfoster care comparison groups on the anxiety, depression (mother comparison only), ADHD-IN, ADHD-HI, LPE (father comparison group only) social impairment, and academic impairment dimensions. Even when the longer stay in foster-care group still had significantly higher scores than the non-foster care comparison groups (i.e., SCT and ODD measures), the magnitude of the difference was much smaller for the longer vs. shorter stay relative to the comparison groups (i.e., SCT: $d$ value of approximately 0.60 vs. 2.00, ODD: $d$ values of approximately 0.70 vs. 1.60 ). 
Longer durations of the home-based foster care in the current study was thus associated with a non-significant difference between the foster-care and the non-foster care comparisons groups on most of the symptom and impairment measures. These findings suggest the foster care duration was associated with an increase in the normalization of behavior of the foster care children. In other words, providing the foster care children with a normal social and emotional context was associated with a reduction in clinical symptoms and impairment.

\section{Limitations and Suggestions for Future Research}

The two main limitations were the small number of children in the foster care sample and the cross-sectional nature of the study. Future research would benefit from a larger sample of children in foster care as well as a broader age range, especially younger children. Future research should also compare children in the complete range of Spanish protective services (i.e., Reception Centers, Host Homes and Extended Family Care) with children in home-based foster care to non-foster care comparison groups. Finally, longitudinal research would provide a useful way to follow-up on the cross-sectional findings of the current study, especially the association of duration of stay in foster care with lower scores on the symptom and impairment dimensions. For example, if a home-based foster care sample were assessed at the start of foster care and at subsequent 4-month intervals through the duration of the foster care with each foster care child matched to a comparison child with assessments at the same time points, then such a study would allow a better understanding of the improvements associated with foster care.

Acknowledgements We wish to thank the families of the Foster Care Program of the Province of Alicante for their invaluable cooperation. We also wish to acknowledge the assistance of the General Direction of Childhood and Adolescence of the Valencian Community whose support made the study possible.

Authors' Contributions INS: Project idea, article concept and design, as well as planning the timeline, substantially involved in the data, material, and article acquisition. Mainly responsible for drafting, writing, and revising the review article, and responsible for selection and final approval of the scholarly publication. MSB: Analyzed the data and wrote part of the results. Provided substantial help with the concept and design. Contributed substantially to the project by drafting and revising the review article and being responsible for $\mathrm{e}$ final approval of the scholarly publication. GLB: Analyzed the data and wrote part of the results. Participated in drafting the work and revising it critically with respect to important intellectual content in all its phases.

\section{Compliance with Ethical Standards}

Conflict of Interest The authors declare that they have no conflict of interest.
Ethical Approval This research was conducted in accordance with the ethical principles that have their origin in the Declaration of Helsinki in its current form. A positive vote of approval regarding the study and its methodology was granted by the institutional review board, the Ethics Committee of the University of Alicante, with the assigned file number being: UA-2018-03-08and Directorate-General of Equality and Inclusive Policies, the agency responsible for children in foster care.

Informed Consent The foster care families signed a written consent form that explained the purpose of the study were also provided with a summary of the findings.

Publisher's note Springer Nature remains neutral with regard to jurisdictional claims in published maps and institutional affiliations.

Open Access This article is distributed under the terms of the Creative Commons Attribution 4.0 International License (http://crea tivecommons.org/licenses/by/4.0/), which permits unrestricted use, distribution, and reproduction in any medium, provided you give appropriate credit to the original author(s) and the source, provide a link to the Creative Commons license, and indicate if changes were made.

\section{References}

Baptista, J., Belsky, J., Marques, S., Silva, J., Oliveira, P., \& Mesquita, A., et al. (2014). The interactive effect of maltreatment in the family and unstable institutional caregiving in predicting behavior problems in toddlers. Child Abuse \& Neglect, 38(12), 2072-2079. https://doi.org/10.1016/j.chiabu.2014.10.015.

Belmar, M., Servera, M., Becker, S. P., \& Burns, G. L. (2017). Validity of sluggish cognitive tempo in South America: an initial examination using mother and teacher ratings of Chilean children. Journal of Attention Disorders, 21, 667-672.

Bernad, M., Servera, M., Becker, S. P., \& Burns, G. L. (2016). Sluggish cognitive tempo and ADHD inattention as predictors of externalizing, internalizing, and impairment domains: a 2-year longitudinal study. Journal of Abnormal Child Psychology, 44, 771-785. https://doi.org/10.1007/s10802-015-0066-z.

Burns, G. L., Lee, S., Servera, M., McBurnett, K., \& Becker, S. P. (2015a). Child and adolescent behavior inventory-parent version 1.0. Pullman, WA: Author.

Burns, G. L., Lee, S., Servera, M., McBurnett, K., \& Becker, S. P. (2015b). Child and adolescent behavior inventory-teacher version 1.0. Pullman, WA: Author.

Burns, G. L., Becker, S. P., Servera, M., Bernad, M., \& García-Banda, G. (2017). Sluggish cognitive tempo and ADHD inattention in the home and school contexts: parent and teacher invariance and cross-setting validity. Psychological Assessment, 29, 209-220.

Del Valle, J. F., Bravo, A., \& López, M. (2009). El acogimientofamiliar en España: implantación y retosactuales. Papelesdel Psicólogo, 30(1), 33-41.

Del Valle, J. F., \& Bravo, A. (2003). Situaciónactualdelacogimientofamiliar en España. Informe de Investigación. Madrid: Ministerio de Trabajo y Asuntos Sociales.

Del Valle, J., López, M., Montserrat, C., \& Bravo, A. (2008). El acogimiento familiar en España. Una evaluación de resultados. Madrid: Ministerio de Trabajo y Asuntos Sociales.

Delgado, L., Fornieles, A., Costas, C., \& Brun-Gasca, C. (2012). Family fostering in Spain. An evaluation of results. Revista de Investigación en Educación (REINED), 10(1), 158-171.

Dozier, M., Zeanah, C. H., Wallin, A. R., \& Shauffer, C. (2012). Institutional care for young children: review of literature and 
policy implications. Social Issues and Policy Review, 6, 1-25. https://doi.org/10.1111/j.1751-2409.2011.01033.x.

Dregan, A., \& Gulliford, M. C. (2012). Foster care, residential care and public care placement patterns are associated with adult life trajectories: population-based cohort study. Social Psychiatry and Psychiatric Epidemiology, 47, 1517-1526. https://doi.org/10. 1007/s00127-011-0458-5.

European Association for Research into Residential Child Care (EUROARRCC). (1998). Care to listen: a report of residential child care in four European countries. Glasgow, Scotland: The Center for Residential Child Care.

Fernández-Molina, M., Del Valle, J., Fuentes, M., Bernedo, I., \& Bravo, A. (2011). Behavioral problems of adolescents in preadoptive, residential and extended family care. Psicothema, 23 (1), 1-6.

Fries, A., Shirtcliff, E., \& Pollak, S. (2008). Neuroendocrine dysregulation following early social deprivation in children. Developmental Psychbilogy, 50(6), 588-599. https://doi.org/10.1002/dev. 20319.

Gil-Lario, M., Ceccato, R., Molero-Mañes, R., \& Ballester-Arnal, R. (2013). Socioemotional characteristics of minors in foster care: a comparison between the estimation of parents, teachers and children. Children and Youth Services Review, 35(4), 664-667. https://doi.org/10.1016/j.childyouth.2013.01.013.

Goemans, A., Tarren-Sweeney, M., Van Geel, M., \& Vedder, P. (2018). Psychosocial screening and monitoring for children in foster care: psychometric properties of the brief assessment checklist in a Dutch population study. Clinical Child Psychology and Psychiatry, 23(1), 9-24. https://doi.org/10.1177/ 1359104517706527.

Gunnar, M. R., \& Van Dulmen, M. H. (2007). Behavior problems in postinstitutionalized internationally adopted children. Development and Psychopathology, 19(1), 129-148. https://doi.org/10. 1017/S095457940707007.

Humphreys, K., Gleason, M., Drury, S., Miron, D., Nelson, C., Fox, N., \& Zeanah, C. (2015). Effects of institutional rearing and foster care on psychopathology at age 12 years in Romania: follow-up of an open, randomised controlled trial. The Lancet Psychiatry, 2 (7), 625-634. https://doi.org/10.1016/s2215-0366(15)00095-4.

Kjelsberg, E., \& Nygren, P. (2004). The prevalence of emotional and behavioural problems in institutionalized childcare clients. Nordic Journal of Psychiatry, 58(4), 319-325. https://doi.org/10.1080/ 08039480410005846.

Li, D., Chng, G. S., \& Chu, C. M. (2017). Comparing long-term placement outcomes f residencial and family foster care: a metaanalysis. Trauma Violence \& Abuse, 1-12. https://doi.org/10. $1177 / 1524838017726427$

López, M., Del Valle, J. F., Montserrat, C., \& Bravo, A. (2010). Niños que esperan. Estudio sobre casos de larga estancia en acogimiento residencial. Madrid: Ministerio de Sanidad y Política Social.

Martín, E., Torbay, A., \& Rodríguez, T. (2008). Family cooperation and bonding of the child with the family in residential fostering programs. Anales de Psicología, 24(1), 25-32.

Muthén, L. K., \& Muthén, B. O. (1998-2017). Mplus user's guide. 8th ed. Los Angeles, CA: Muthén \& Muthén.
Palacios, J. (2003). Institutions for children: protection or risk? Infancia y Aprendizaje, 26(3), 353-363. https://doi.org/10.1174/ 021037003322299098 .

Pinheiro, P. S. (2006). United Nations secretary-general's study on violence against children. Geneva, Switzerland: ATAR-Roto Presse SA. https://www.unicef.org/lac/full_tex(3).pdf.

Rutter, M., Sonuga-Barke, E., \& Castle, J. (2010). I. Investigating the impact of early institutional deprivation on development: background and research strategy of the English and Romanian adoptees (ERA) study. Monographs of the Society for Research in Child Development, 75(1), 1-20. https://doi.org/10.1111/j. 1540-5834.2010.00548.x.

Servera, M., Bernad, M. M., Carrillo, J. M., Collado, S., \& Burns, G. L. (2016). Longitudinal correlates of sluggish cognitive tempo and ADHD-inattention symptom dimensions with Spanish children. Journal of Clinical Child and Adolescent Psychology, 45, 632-641.

Sawyer, M. G., Carbone, J. A., Searle, A. K., \& Robinson, P. (2007). The mental health and wellbeing of children and adolescents in homebased foster care. Medical Journal of Australia, 186, 181-184.

Sáez, B., Servera, M., Becker, S. P. \& Burns, G. L. (2018). Optimal items for assessing sluggish cognitive tempo in children across mother, father, and teacher ratings. Journal of Clinical Child and Adolescent Psychology. https://doi.org/10.1080/15374416.2017. 1416619. (Advance of Print).

Seijas, R., Servera, M., Garcia-Banda, G., Barry, C., \& Burns, G. L. (2018a). Evaluation of a four-item DSM-5 limited prosocial emotions specifier scale within and across settings with Spanish children. Psychological Assessment, 30, 474-485.

Seijas, R., Servera, M., Garcia-Banda, G., Burns, G. L., Preszler, J., Barry, C., Litson, K., \& Geiser, C. (2018b). Consistency of limited prosocial emotions across occasions, sources, and settings: Trait- or state-like construct in a young community sample? Journal of Abnormal Child Psychology, 47(1), 47-58.

Shechory, M., \& Sommerfeld, E. (2007). Attachment style, homeleaving age and behavioral problems among residential care children. Chld Psychiatry and Human Development, 37(4), 361-373. https://doi.org/10.1007/s10578-007-0051-z.

Smyke, A. T., Zeanah, C. H., Gleason, M. M., Drury, S. S., Fox, N. A., Nelson, C. A., \& Guthrie, D. (2012). A randomized controlled trial comparing foster care and institutional care for children with signs of reactive attachment disorder. American Journal of Psychiatry, 169, 508-514. https://doi.org/10.1176/appi.ajp.2011. 11050748 .

Tarren-Sweeney, M. (2017). Mental health services for our most vulnerable children. Clinical Child Psychology and Psychiatry, 22(4), 521-523. https://doi.org/10.1177/1359104517734093.

Tibu, F., Humphreys, K., Fox, N., Nelson, C., \& Zeanah, C. (2014). Psychopathology in young children in two types of foster care following institutional rearing. Infant Mental Health Journal, 35 (2), 123-131. https://doi.org/10.1002/imhj.21428.

Troller-Renfree, S., Zeanah, C. H., Nelson, C. A., \& Fox, N. A. (2018). Neural and cognitive factors influencing the emergence of psychopathology: insights from the Bucharest Early Intervention Project. Child Development Perspectives, 12, 28-33. https://doi. org/10.1111/cdep.12251. 